\title{
First-Principles Study of Structural, Electronic, Magnetic, Optical, and Magneto-Optical Properties of NpN
}

\author{
Shugo Suzuki, Ming-Fang LI, and Toshihiro Ariizumi \\ Institute of Materials Science, University of Tsukuba, Tsukuba 305-8573
}

(Received April 25, 2008)

\begin{abstract}
We study the structural, electronic, magnetic, optical, and magneto-optical properties of $\mathrm{NpN}$ in detail using the fully relativistic full-potential calculations based on the density functional theory within the local spin density approximation. We successfully reproduce the positive sign of the electric field gradient (EFG) at Np nuclei. The positive EFG is in striking contrast to the negative $\mathrm{EFG}$ in a $\mathrm{Np}^{3+}$ free ion, indicating the importance of the interplay between spin-orbit coupling and covalent bonding in NpN. Also, the calculated band structure shows large splittings induced by spin polarization and spin-orbit coupling, suggesting that both effects are indispensable for understanding the electronic properties of this material. This results in a large Kerr rotation angle of about 2 degrees, which is comparable to those of uranium calcogenides.
\end{abstract}

KEYWORDS: NpN, density functional theory, local spin density approximation, fully relativistic calculation, full-potential calculation, structural properties, electronic properties, magnetic properties, optical properties, magneto-optical properties

\section{Introduction}

Physics of actinide compounds has been one of the important research fields of condensed matter physics for over a half century, particularly in connection with the strong spin-orbit coupling associated with actinide atoms. ${ }^{1)}$ Among the compounds, those with the rock-salt structure $\operatorname{An} X$, where $\mathrm{An}$ is an actinide element and $X$ is an anion element, are regarded as a representative and their properties have been studied both experimentally and theoretically. $^{2-27)}$ Despite the simpleness of the structure, $A n X$ shows a wide variety of magnetic phenomena, such as simple ferromagnetic to complex antiferromagnetic structure, ${ }^{2,3,11,27)}$ the dominance of orbital magnetic moment over spin magnetic moment, $, 5,6,9,14,16,19,20,23,27)$ and a large magneto-optical effect. ${ }^{11,13,14,17)}$

Thus far, several theoretical studies of $A n X$ have been carried out to elucidate trends upon replacing the constituent atoms and have successfully explained the overall properties. $^{4,6-10,12,22,25)}$ For example, a series of studies performed by Brooks made very important contributions to the understanding of the trends in the structural and electronic properties of $\mathrm{An} X{ }^{4,6-8,10)}$ In these studies, performing the calculations based on the density functional theory within the local density approximation (LSDA), the lattice constants and the bulk 
moduli were studied using an approximate equation of state and also the band structures were calculated assuming paramagnetic ground states. Despite the several approximations employed in these studies, the overall properties have been explained successfully.

On the other hand, there are not so many detailed theoretical studies of individual $\operatorname{An} X$. The compounds relatively studied in detail are uranium pnictides and calcogenides, $\mathrm{U} X$, where $X=\mathrm{N}, \mathrm{P}, \mathrm{As}, \mathrm{Sb}, \mathrm{S}, \mathrm{Se}$, and Te. ${ }^{5,13-17,19,20,23,26)}$ One of the possible reasons why UX has been studied in detail is that most $\mathrm{U} X$ compounds are ferromagnetic and have one formula unit in each unit cell, and accordingly, theoretical studies of such compounds are relatively easy. Firstly, an important study was carried out by Brooks and Kelly.5) In this study, it has been shown that a large orbital magnetic moment dominates the spin magnetic moment and plays an essential role in magnetic properties of $\mathrm{UX}$. Also, for the structural properties, a detailed study employing the full-potential linear muffin-tin-orbital method within the LSDA was carried out. ${ }^{15)}$ In this study, the previous results obtained using an approximate equation of state were reproduced by performing more elaborate calculations. Furthermore, for the electronic, magnetic, optical, and magneto-optical properties, several detailed studies have been carried out within the LSDA and the muffin-tin potential approximation. ${ }^{13,14,17)}$ In these studies, Kerr spectra were calculated, and as a result, a large Kerr rotation angle of about 3 degrees was found to be in agreement with the experimental result, although the detailed structures of the calculated spectra were not found to agree with each other and the experimentally observed one. In addition, there is a recent study involving the X-ray magneticcircular-dichroism (XMCD) spectra of uranium calcogenides. ${ }^{23}$ ) This seems important because the analysis of the XMCD spectra enables us to investigate the orbital magnetic moment of the actinide atoms using the XMCD sum rule. ${ }^{28,29)}$ The properties of $\mathrm{U} X$ have thus been studied theoretically in detail to a satisfactory extent.

On the contrary, there are very few detailed theoretical studies of $\mathrm{Np} X$, where $X=\mathrm{N}, \mathrm{P}$, As, and Sb. Among recently published papers, we can find only one theoretical study. ${ }^{27)}$ In this study, Atta-Fynn and Ray have investigated the structural and electronic properties of NpN, as well as those of other actinide nitrides, using the full-potential linearized augmented plane wave plus local orbital method. They have shown that the total energy significantly decreases with the inclusion of spin-orbit coupling, implying that the effect of spin-orbit coupling is large and cannot be ignored. However, in their study, spin-orbit coupling was treated perturbatively, and if possible, it is desirable to treat spin-orbit coupling more accurately, i.e., by solving the Dirac equation directly. Moreover, $\mathrm{Np} X$ is worth being studied in detail because of the following reason. Since ${ }^{237} \mathrm{~Np}$ nuclei possess a non-zero electric quadrupole moment, ${ }^{30)}$ the electric field gradient (EFG) at the Np nuclei can be measured experimentally by, e.g., Mössbauer spectroscopy. ${ }^{2}$ In particular, for $\mathrm{Np} X$, the study of the EFG at the Np nuclei is interesting owing to the following reason. Since the rock-salt structure is cubic, the EFG at the Np nuclei 
in $\mathrm{Np} X$ might, at first sight, be expected to be zero. It is not, however, the case; the charge distribution around a $\mathrm{Np}$ nucleus does not show the cubic symmetry purely because of the existence of a non-zero orbital magnetic moment due to the strong spin-orbit coupling. To calculate the EFG in $\mathrm{Np} X$, it is indispensable to employ not only fully relativistic but also full-potential calculations. However, this type of calculation has not been carried out yet for studying the EFG in $\mathrm{Np} X$. It is then natural to begin with the simplest compound of $\mathrm{Np} X$, i.e., NpN, which is ferromagnetic and has one unit formula in the unit cell.

In this study, we investigate the structural, electronic, magnetic, optical, and magnetooptical properties of $\mathrm{NpN}$ using the fully relativistic full-potential calculations based on the density functional theory within the LSDA. To examine the reliability of the results of our calculations with respect to the quality of basis sets, we employ two methods: the fully relativistic full-potential linear-combination-of-atomic-orbitals (FFLCAO) method and the fully relativistic full-potential mixed-basis (FFMB) method. ${ }^{31)}$ In $\S 2$, we describe the method of calculations briefly. Next, $\S 3$ is devoted to the results and discussion. In this section, we present the structural, electronic, magnetic, optical, and magneto-optical properties of $\mathrm{NpN}$ in detail. We show the results of the optimization of the lattice constant and then the results of the calculations of the cohesive energy and bulk modulus. The orbital and spin magnetic moments are studied next and the results of the calculations of the EFG are examined. Also, the band structure and the densities of states are given. Furthermore, the results of calculating the optical conductivity tensor, reflectivity, Kerr and XMCD spectra are studied. In particular, we compare the orbital magnetic moment calculated using a conventional method with that calculated using the XMCD sum rule. Finally, the conclusions of this study are given in $\S 4$.

\section{Method of Calculations}

NpN crystallizes in the rock-salt structure. ${ }^{2)}$ The lattice constant in the equilibrium structure at ambient pressure is $4.90 \AA$. In the ferromagnetic phase, the magnetization is parallel to the (111) axis. In this study, we consider the (111) axis as the $z$-axis. It should be noted that the crystal still remains in the rock salt structure because all the atomic coordinates and primitive vectors are rotated simultaneously. Also, to compare the results of calculations with the available experimental data, all the calculations presented in this study were carried out using the experimental lattice constant except for the optimization of the lattice constant itself and the calculations of the cohesive energy and bulk modulus because these quantities themselves are the structural properties with respect to the most stable structure. We employed the FFLCAO and FFMB methods. ${ }^{31)}$ Using the two methods, we can examine the reliability of the results of calculations with respect to the quality of basis sets because two basis sets are distinctly different from each other. In both methods, we deal exactly with all relativistic effects including spin-orbit coupling by solving Dirac-Kohn-Sham equations directly. It should be noted that, in the FFLCAO and FFMB methods, all the matrix elements of the Hamilto- 
nian of the Dirac-Kohn-Sham equations are calculated using four-component basis functions, and as a result, spin-orbit coupling, as well as other relativistic effects, is considered without any approximation except that the number of basis functions is finite. Also, by spherical harmonic expansion, we calculated the electrostatic potential without any shape approximation, thereby calculating the EFG at the Np nuclei accurately. We expanded the electrostatic potential up to $l=8$, where $l$ denotes the degree of spherical harmonic expansion. Furthermore, using the fully relativistic expression for the optical conductivity tensor, where the matrix elements of the Dirac matrices are used as the transition matrix elements instead of those of the momentum operator, ${ }^{32)}$ the Kerr and XMCD spectra were highly precisely calculated. In all calculations, we employed the LSDA exchange-correlation potential represented by the Perdew-Zunger parameterization of Ceperly-Alder results. ${ }^{35,36)}$

The four-component atomic orbitals used in the FFLCAO method are as follows: $1 s, 2 s$, $2 p, 3 s, 3 p, 3 d, 4 s, 4 p, 4 d, 4 f, 5 s, 5 p, 5 d, 5 f, 6 s, 6 p, 6 d$, and $7 s$ orbitals of neutral Np atoms, $5 f, 7 s$, and $7 p$ orbitals of $\mathrm{Np}^{2+}$ atoms, $1 s, 2 s$, and $2 p$ atomic orbitals of neutral $\mathrm{N}$ atoms, and $2 s$ and $2 p$ orbitals of $\mathrm{N}^{2+}$ atoms, and $3 d$ orbitals of $\mathrm{N}^{5+}$ atoms. It is necessary to use not only the atomic orbitals of neutral atoms but also those of positively charged atoms to describe the contraction of atomic orbitals accompanied by cohesion. On the other hand, the basis function adopted in the FFMB method consists of the four-component atomic orbitals of neutral $\mathrm{Np}$ and $\mathrm{N}$ atoms used in the FFLCAO method, and four-component plane waves, which are positive-energy solutions of the Dirac equation for a free electron. In this study, we chose the cut-off energy of the four-component plane waves to be $50 \mathrm{eV}$. This cut-off energy corresponds to 26 four-component plane waves for each $\boldsymbol{k}$ point.

We carried out real-space integration using 4644 points for the Np atom and 2064 points for the $\mathrm{N}$ atom. Also, we carried out Brillouin-zone integration using two methods: the specialpoint method for optimizing the lattice constant and the good-lattice-point method for calculating the electronic, magnetic, optical, and magneto-optical properties. ${ }^{37,38)}$ In optimizing the lattice constant, we used $64 \boldsymbol{k}$ points; we confirmed the convergence of the total energy with respect to the number of $\boldsymbol{k}$ points and found it to be less than $0.001 \mathrm{eV} /$ atom. The cohesive energy was calculated for several lattice constants and then the bulk modulus was evaluated using Murnaghan's equation of state. ${ }^{39)}$ In calculating the electronic, magnetic, optical, and magneto-optical properties, we used up to $1958 \boldsymbol{k}$ points by increasing the number of $\boldsymbol{k}$ points to confirm the convergence.

We also studied the spin and orbital magnetic moments. It should, however, be noted that the calculation of orbital magnetic moments can be carried out only using a method that employs local orbitals as basis functions. ${ }^{40}$ ) At present, there are no formulas available for calculating orbital magnetic moments when plane waves are used as basis functions as in the FFMB method. For this reason, we calculated the orbital magnetic moment only using the 
FFLCAO method. Moreover, a non-zero orbital magnetic moment results in a non-zero EFG at the Np nuclei, which was observed experimentally by Mössbauer measurement. ${ }^{2)}$ The EFG is given by

$$
V_{\xi \eta}=\frac{\partial^{2} V}{\partial \xi \partial \eta}
$$

where $V$ represents the electrostatic potential and $\xi$ and $\eta$ denote Cartesian coordinates; ${ }^{41)}$ the second derivative on the right-hand side is evaluated at the Np nuclei. By diagonalizing $V_{\xi \eta}$, we obtain three eigenvalues, $V_{x x}, V_{y y}$, and $V_{z z}$. Customarily, they are arranged in the order such that $\left|V_{x x}\right| \leq\left|V_{y y}\right| \leq\left|V_{z z}\right|$. The coupling constant $e^{2} q Q / h$ is then defined by

$$
e^{2} q Q / h=e V_{z z} Q / h
$$

where $Q$ represents the electric quadrupole moment of a nucleus; we used $Q=+3.886$ barn for the ${ }^{237} \mathrm{~Np}$ nuclei. ${ }^{30)}$ Also, the asymmetry parameter is defined by $\left(V_{x x}-V_{y y}\right) / V_{z z}$. This quantity in $\mathrm{NpN}$, however, is zero because the axial symmetry still survives even when there exists a non-zero orbital magnetic moment. Furthermore, in calculating the optical conductivity tensor $\sigma_{\xi \eta}$ as a function of photon energy $\hbar \omega$, we used the fully relativistic expression, where the matrix elements of the Dirac matrices $\boldsymbol{\alpha}$, instead of those of the momentum operator, are used as the transition matrix elements between the occupied state $|\boldsymbol{l} \boldsymbol{k}\rangle$ and the unoccupied state $|n \boldsymbol{k}\rangle:^{32)}$

$$
\begin{aligned}
\sigma_{\xi \eta}= & \frac{2 i e^{2} c^{2}}{\hbar \Omega} \sum_{\boldsymbol{k}} \sum_{l} \sum_{n} \frac{f_{l \boldsymbol{k}}\left(1-f_{n \boldsymbol{k}}\right)}{\omega_{\tau}^{2}-\omega_{n l \boldsymbol{k}}^{2}} \\
& \times\left\{\frac{\omega_{\tau}}{\omega_{n l \boldsymbol{k}}} \operatorname{Re}\left[\left\langle l \boldsymbol{k}\left|\alpha_{\xi}\right| n \boldsymbol{k}\right\rangle\left\langle n \boldsymbol{k}\left|\alpha_{\eta}\right| l \boldsymbol{k}\right\rangle\right]+i \operatorname{Im}\left[\left\langle l \boldsymbol{k}\left|\alpha_{\xi}\right| n \boldsymbol{k}\right\rangle\left\langle n \boldsymbol{k}\left|\alpha_{\eta}\right| l \boldsymbol{k}\right\rangle\right]\right\},
\end{aligned}
$$

where $\omega_{n l \boldsymbol{k}}$ denotes the energy difference between the occupied and unoccupied states, $\left(\varepsilon_{n \boldsymbol{k}}-\right.$ $\left.\varepsilon_{l \boldsymbol{k}}\right) / \hbar$, and $\omega_{\tau}$ denotes $\omega+\hbar / \tau$ with $\tau$ being the lifetime of excited electrons. Also, $\Omega$ denotes the volume of the unit cell and $f_{l \boldsymbol{k}}$ and $f_{n \boldsymbol{k}}$ denote occupation numbers. Here, we consider only the interband contribution to the optical conductivity tensor to avoid an ambiguity due to the phenomenological description of the intraband contribution by the Drude formula. Then the reflectivity and Kerr spectra were calculated using the optical conductivity tensor. The reflectivity $R_{ \pm}$, where the plus and minus signs refer to the left and right circular polarizations, respectively, was calculated using

$$
R_{ \pm}=\frac{\left(n_{ \pm}-1\right)^{2}+\kappa_{ \pm}^{2}}{\left(n_{ \pm}+1\right)^{2}+\kappa_{ \pm}^{2}}
$$

In this equation, $n_{ \pm}$and $\kappa_{ \pm}$are the real and imaginary parts of the complex refractive index, respectively.

$$
n_{ \pm}+i \kappa_{ \pm}=\sqrt{\varepsilon_{x x} \pm i \varepsilon_{x y}}
$$

Here, $\varepsilon_{\xi \eta}$ is the dielectric tensor defined by $\varepsilon_{\xi \eta}=\delta_{\xi \eta}+4 \pi i \sigma_{\xi \eta} / \omega$. Also, the Kerr rotation $\theta_{\mathrm{K}}$ 
and Kerr ellipticity $\eta_{\mathrm{K}}$ were calculated using

$$
\theta_{\mathrm{K}}+i \eta_{\mathrm{K}}=-\frac{\sigma_{x y}}{\sigma_{x x} \sqrt{1+i(4 \pi / \omega) \sigma_{x x}}} .
$$

Finally, we explain the method for calculating the XMCD. The fully relativistic calculations of the XMCD have been presented in previous pioneering works. ${ }^{33,34)}$ Although the essence of the method is the same, we calculated the XMCD using the imaginary part of the complex refractive index $\kappa_{ \pm}$; the total absorption coefficient $\mu_{\text {tot }}$ and the XMCD $\Delta \mu$ of the $M_{4,5}$ edges of $\mathrm{Np}$ are given by

$$
\mu_{\mathrm{tot}}=\frac{2 \omega}{c}\left(\kappa_{+}+\kappa_{-}\right)
$$

and

$$
\Delta \mu=\frac{2 \omega}{c}\left(\kappa_{+}-\kappa_{-}\right),
$$

respectively. In addition, the absorption coefficient for light linearly polarized along the $z$ axis,

$$
\mu_{z}=\frac{2 \omega}{c} \kappa_{z}
$$

where $\kappa_{z}=\operatorname{Im} \sqrt{\varepsilon_{z z}}$, was used for calculating the orbital moment using the XMCD sum rule. ${ }^{28,29)}$ In the analysis of the results presented in the next section, we used the lifetime parameter $\hbar / \tau=0.5 \mathrm{eV}$ for the infrared to ultraviolet region, while we used $\hbar / \tau=2.5 \mathrm{eV}$ for the $\mathrm{X}$-ray region.

\section{Results and discussion}

We begin with the results of the optimization of the lattice constant. In Table I, we show the optimized lattice constant, as well as the cohesive energy, and the bulk modulus calculated using the optimized lattice constant. We also show those obtained by experiments in the table. The lattice constant optimized using the FFLCAO (FFMB) method is 4.77 (4.78) $\AA$; the error is found to be $-2.7(-2.4) \%$ with respect to the experimental lattice constant of $4.90 \AA$. This error is larger than those known for well-studied materials, such as diamond and Si. However, this error is acceptable when the LSDA is used. As will be shown when discussing the results of the calculations of the band structure, the bonding between the $\mathrm{Np}$ and $\mathrm{N}$ atoms is covalent to some degree. The underestimation in optimizing the lattice constant of $\mathrm{NpN}$ may be due to the overestimation of the covalent bonding between the Np-5f and $\mathrm{N}-2 p$ orbitals. This is mainly because the incorporation of the electron correlation between the Np- $5 f$ electrons by the LSDA is not perfect. Nevertheless, it thus seems that we can deal with the $5 f$ electrons in $\mathrm{NpN}$ as itinerant electrons using the LSDA with acceptable errors accompanied by this approximation.

The optimization of the lattice constant of $\mathrm{NpN}$ was successfully carried out in a pioneering study by Brooks assuming a paramagnetic ground state. ${ }^{8)}$ In his study, the following equation 
of state describing the electronic pressure $P$ was employed.

$$
P=-\frac{\delta}{\delta V}\left(\sum_{i} n_{i} \varepsilon_{i}\right)-\sum_{t}\left(\frac{1}{4 \pi S_{t}^{2}}\right) \int_{S_{t}} n\left(S_{t}\right)\left\{\varepsilon_{\mathrm{xc}}\left(S_{t}\right)-\mu_{\mathrm{xc}}\left(S_{t}\right)\right\} \mathrm{d} S_{t}+U_{\mathrm{M}}
$$

Here, $\varepsilon_{\mathrm{xc}}\left(S_{t}\right)$ and $\mu_{\mathrm{xc}}\left(S_{t}\right)$ represent the exchange-correlation energy and potential, respectively, $\varepsilon_{i}$ is the eigenvalue, $n(r)$ the charge density, $S_{t}$ the Wigner-Seitz radius of atom $t$ in the primitive cell, $n\left(S_{t}\right)$ the charge density at $S_{t}$, and $U_{\mathrm{M}}$ the Madelung term. Using this approximate equation of state, Brooks determined the optimized lattice constant to be 4.95 $\AA$, which is in very good agreement with the experimental lattice constant of $4.90 \AA$, with an overestimation of about $+1 \%$. Although this overestimation, which is in contrast to the underestimation found in our study, may be caused by the approximations employed when deriving the equation of state or the assumption of a paramagnetic ground state or both, the excellent agreement indicates that the previous calculations have successfully captured the essential features of the elastic properties of NpN. Atta-Fynn and Ray have carried out the optimization of the lattice constant of $\mathrm{NpN}$ in a recent study. ${ }^{27)}$ The optimized lattice constant was found to be $4.86 \mathrm{~A}$ with an underestimation of about $-1 \%$. Although they treated spin-orbit coupling perturbatively, the result is in very good agreement with the experimental lattice constant.

The cohesive energy calculated using the FFLCAO (FFMB) method is 7.99 (8.04) $\mathrm{eV} /$ atom when the optimized lattice constant is used. Although not shown in the table, when the experimental lattice constant is used, the cohesive energy calculated using the FFLCAO (FFMB) method is 7.91 (7.98) eV/atom. In both cases, the calculated cohesive energy is overestimated by about $2 \mathrm{eV} /$ atom in comparison with the experimental one, $6.04 \mathrm{eV} /$ atom. ${ }^{18}$ ) The overestimation often occurs when using the LSDA. For example, the LSDA overestimates the cohesive energy of graphite by about $1.6 \mathrm{eV} .{ }^{42)}$ We may thus conclude that the overestimation of the cohesive energy of about $2 \mathrm{eV}$ is acceptable when the LSDA is used. The bulk modulus calculated using the FFLCAO (FFMB) method is 230 (250) GPa. The previous result, $200 \mathrm{GPa}$, which is obtained using the approximate equation of state, ${ }^{8)}$ is smaller than our results by about $20 \%$. Although the experimental bulk modulus has not been measured yet, we believe that our results are also in agreement with the experimental bulk modulus with an error of about $20 \%$, which is a typical error associated with the use of the LSDA in calculating the bulk modulus. Atta-Fynn and Ray calculated the cohesive energy and the bulk modulus of NpN. ${ }^{27)}$ They obtained $6.5 \mathrm{eV} /$ atom for the cohesive energy and $183 \mathrm{GPa}$ for the bulk modulus. Both results are considerably smaller than our results. There are several possible origins for these discrepancies. One possible origin is that Atta-Fynn and Ray treated spin-orbit coupling perturbatively, not by solving the Dirac equation directly, and thus, the total energy might not be calculated accurately. Another possible origin is that they adopted the generalized gradient approximation, which typically gives larger lattice constants 
and smaller cohesive energies, as well as smaller bulk moduli, than those in the case of using the LSDA. However, to elucidate the origin of the discrepancies, further theoretical studies are necessary.

We next examine the results of the calculations of the spin and orbital magnetic moments per formula unit. The non-zero orbital magnetic moment is the direct consequence of spinorbit coupling. The results of calculations are shown in Table II together with those obtained by experiments. The calculations were carried out using the experimental lattice constant to compare the results of calculations with those of experiments. Here, we mention again that we cannot calculate the orbital magnetic moment using the FFMB method because there are no formulas available for calculating orbital magnetic moments when plane waves are used as basis functions. It is found that the spin and orbital magnetic moments are antiparallel to each other. Furthermore, the magnitude of the orbital magnetic moment calculated using the FFLCAO method, $2.55 \mu_{\mathrm{B}}$, is larger than that of the spin magnetic moment calculated using the FFLCAO (FFMB) method, $-2.09(-2.20) \mu_{\mathrm{B}}$, as is also known for US for which the magnitude of the calculated orbital magnetic moment, $2.43 \mu_{\mathrm{B}}$, is larger than that of the calculated spin magnetic moment, $-1.66 \mu_{\mathrm{B}} \cdot{ }^{14)}$ The calculated total magnetic moment, $0.46 \mu_{\mathrm{B}}$, is, however, considerably smaller than the experimental total magnetic moment, $1.38 \mu_{\mathrm{B}}{ }^{2)}$ The underestimation of the total magnetic moment was also found in US; the calculated total magnetic moment of US is $0.8 \mu_{\mathrm{B}}$, while the experimental total magnetic moment is 1.6-1.7 $\mu_{\mathrm{B}} \cdot{ }^{14)}$ The underestimation may be caused by the deficiency of the LSDA, as pointed out in the previous studies. ${ }^{5,6,9,14,23)}$ Atta-Fynn and Ray calculated the total spin magnetic moment to be $-2.45 \mu_{\mathrm{B}} \cdot{ }^{27)}$ Their result is larger than our result. Atta-Fynn and Ray also calculated the site-projected spin, orbital, and total magnetic moments to be $-2.26,2.21,-0.05 \mu_{\mathrm{B}}$, respectively. These results also support the cancellation of the spin and orbital magnetic moments. However, the spin magnetic moment slightly dominates the orbital magnetic moment in contrast to our result. The origin of the discrepancy might be due to the hypothetical magnetization axis assumed by Atta-Fynn and Ray; they chose the quantization axis for magnetization to be in the (001) direction, not in the experimentally observed direction, i.e., the (111) direction, which we consider as the magnetization axis in our calculations. To elucidate whether the spin magnetic moment dominates the orbital magnetic moment, further theoretical and experimental studies are indispensable.

Furthermore, a quantity closely related to the orbital magnetic moment or spin-orbit coupling in NpN is the EFG at the Np nuclei. Since the crystal structure of NpN is cubic, the charge density, at first sight, might be expected to have a cubic symmetry, and accordingly, the EFG to be zero. In NpN, however, this is not the case; the charge density does not show the cubic symmetry with respect to the Np nucleus purely because of the existence of a non-zero orbital magnetic moment or spin-orbit coupling. Consequently, the non-zero EFG arises at 
Table I. Lattice constant $(\AA)$, cohesive energy (eV/atom), and bulk modulus (GPa). The optimized lattice constant is used for the calculations of the cohesive energy and bulk modulus.

\begin{tabular}{lccc}
\hline & $\begin{array}{c}\text { Lattice } \\
\text { constant }\end{array}$ & $\begin{array}{c}\text { Cohesive } \\
\text { energy }\end{array}$ & $\begin{array}{c}\text { Bulk } \\
\text { modulus }\end{array}$ \\
\hline FFLCAO $^{\text {a) }}$ & 4.77 & 7.99 & 230 \\
FFMB $^{\text {b) }}$ & 4.78 & 8.04 & 250 \\
Expt. & $4.90^{\mathrm{c})}$ & $6.04^{\mathrm{d})}$ & - \\
\hline
\end{tabular}

a) Fully relativistic full-potential LCAO calculations

b) Fully relativistic full-potential mixed-basis calculations

c) Ref. 2

d) Ref. 18

the Np nuclei. It should be noted that the EFG vanishes if one calculates this quantity using the electrostatic potential spherically symmetric with respect to the nuclear position, as in the case of the muffin-tin potential approximation. Accordingly, the full-potential calculations are indispensable in the study of the EFG. The EFG calculated using the FFLCAO (FFMB) method is $+96(+107) \mathrm{MHz} /$ barn with the principal axis along the $z$-axis. The experimental $e^{2} q Q / h$ is $+218 \mathrm{MHz}$, and accordingly, the experimental EFG can be estimated to be +56 $\mathrm{MHz} /$ barn if we use +3.886 barn for the nuclear quadrupole moment of ${ }^{237} \mathrm{~Np}^{2,30}$ ) It should be noted that at least the positive sign and the order of magnitude of the EFG are successfully reproduced, although the errors are large. The reproduction of the positive sign seems important because the sign of the EFG in a $\mathrm{Np}^{3+}$ free ion is negative. ${ }^{2)}$ We calculated the EFG in a $\mathrm{Np}^{3+}$ free ion and found it to be $-350 \mathrm{MHz} /$ barn. This means that the positive sign of the EFG originates in the interplay between spin-orbit coupling and the solid state effect. It is most likely that the strong covalent bonding between $\mathrm{Np}$ and $\mathrm{N}$ atoms overcomes spin-orbit coupling and results in a positive EFG.

Here, it might be worth discussing the reason why the calculation of the EFG is successful, although the calculations of magnetic moments are not very satisfactory. It has been established that the LSDA describes the structural properties well even if it does not describe the magnetic properties very satisfactorily. This means that the LSDA can give a realistic electronic charge density, although it gives a poor description of the spin density. Accordingly, the EFG can be calculated satisfactorily using the LSDA because the EFG originates from the electronic charge density. Therefore, we believe that the result of our calculations that the EFG is positive is reliable, although the calculations of magnetic moments are not very satisfactory.

We now show the band structure obtained in this study in Fig. 1. The band structure 
Table II. Spin, orbital, and total magnetic moments ( $\mu_{\mathrm{B}} /$ formula unit), and electric field gradient (MHz/barn). The experimental lattice constant is used for the calculations.

\begin{tabular}{lrrcc}
\hline & $M_{\text {spin }}$ & $M_{\text {orb }}$ & $M_{\text {tot }}$ & EFG \\
\hline FFLCAO $^{\mathrm{a})}$ & -2.09 & 2.55 & 0.46 & +96 \\
FFMB $^{\mathrm{b})}$ & -2.20 & - & - & +107 \\
Expt. & - & - & $1.38^{\mathrm{c})}$ & $+56^{\mathrm{d})}$ \\
\hline
\end{tabular}

a) Fully relativistic full-potential LCAO calculations

b) Fully relativistic full-potential mixed-basis calculations

c) Ref. 2

d) The experimental electric field gradient is calculated, assuming +3.886 barn for the nuclear quadrupole moment of ${ }^{237} \mathrm{~Np}$, as reported in ref. 30 .

obtained using the FFLCAO method and that obtained using the FFMB method agree very well with each other, and hence, we show only the result of the FFLCAO calculations; the differences between the one-electron energies obtained using the FFLCAO method and those obtained using the FFMB method are found to be less than $0.1 \mathrm{eV}$ in the energy range shown in Fig. 1. In this figure, it should be noted that up-spin and down-spin bands can no longer be distinguished in a strict sense because of spin-orbit coupling, and accordingly, they are shown together. The six bands between -6 and $-1 \mathrm{eV}$ consist mainly of $\mathrm{N}-2 p$ orbitals, and thus, they can be referred to as the N-2p bands, although they hybridize considerably with the Np-5 $f$, Np-6d, and Np-7s orbitals. Furthermore, the fourteen bands between -2 and $2 \mathrm{eV}$ consist mainly of Np-5f orbitals. Above $2 \mathrm{eV}$, there exist bands with large dispersions; these bands consist of Np-6d and Np-7s orbitals. Although not shown in the figure, it is found that the Np-6 $p$ and N-2s orbitals form hybridized bands with a considerable dispersion at about -15 $\mathrm{eV}$. This suggests that the Np-6p orbitals should be dealt with as valence orbitals, as is the case of materials with light actinide atoms. ${ }^{43)}$ The band structure of $\mathrm{NpN}$ was calculated using the relativistic linear muffin-tin orbital method assuming a paramagnetic ground state. ${ }^{8)}$ As a result, there is a notable difference between the band structure obtained in this study and that obtained in the previous study. The bands calculated in this study are split owing to the exchange interaction. In particular, this is significant for the Np bands. For example, at the $\Gamma$ point, the two bands just below the Fermi level show a split of about $0.7 \mathrm{eV}$.

In Figs. 2(a)-2(d), we show the spin-projected densities of states (SPDOS) per formula unit calculated using the FFLCAO method. The energy range is the same as that in Fig. 1. Shown in Fig. 2(a) is the total SPDOS, while shown in Figs. 2(b)-2(d) are the partial SPDOSs for Np- $5 f, \mathrm{~Np}-6 d$ and Np-7s, and N-2p orbitals, respectively. It should be noted that the scale of the vertical axes shown in Figs. 2(a) and 2(b) is ten times larger than that shown in Figs. 
$2(\mathrm{c})$ and 2(d). It is found that the electronic states near the Fermi level consist mainly of Np- $5 f$ orbitals. It is also found that the $\mathrm{N}-2 p$ orbitals contribute between -6 and $-1 \mathrm{eV}$, with a considerable hybridization with $\mathrm{Np}-5 f, \mathrm{~Np}-6 d$, and $\mathrm{Np}-7 s$ orbitals. This means that the bonding between $\mathrm{N}$ and $\mathrm{Np}$ atoms is covalent to some degree. At the same time, the bonding between the $\mathrm{N}$ and $\mathrm{Np}$ atoms is also ionic because all the $\mathrm{N}-2 p$ bands are completely filled, and thus, the charge transfer from $\mathrm{Np}$ atoms to $\mathrm{N}$ atoms must occur. The partial density of states calculated by Atta-Fynn and Ray has also shown that the N-2p states hybridize considerably with the Np-5f and Np-6d states. At the same time, they have confirmed that the charge accumulation around $\mathrm{N}$ atoms and the charge depletion around $\mathrm{Np}$ atoms occur, and concluded that the bonding is also ionic, although, as noted in their paper, this does not necessarily mean the lack of covalency in the bonding between $\mathrm{N}$ and $\mathrm{Np}$ atoms.

We next show the results of the calculations of the optical conductivity tensor. In Figs. 3(a) and 3(b), the calculated real and imaginary parts of the diagonal optical conductivity $\sigma_{x x}$ are shown, respectively. Solid lines represent the results of the FFLCAO calculations, and dotted lines, the results of the FFMB calculations; the agreement is found to be satisfactory. A broad peak is found in the real part around 3-6 eV with a height of about $5 \times 10^{-15} \mathrm{~s}^{-1}$, which is almost the same as the typical height of peaks in the calculated real parts of Fe and US reported previously. ${ }^{14,17,32,44)}$ A corresponding structure is also found in the imaginary part around 3-6 eV. Analyzing the origin of transitions, we found that the broad peak around 3-6 $\mathrm{eV}$ originates in two transitions, namely, the Np-5f $\rightarrow \mathrm{Np}-6 d$ and N-2p $\rightarrow \mathrm{Np}-5 f$ transitions. The former is the intraatomic transition in Np atoms, while the latter is the charge transfer transition between $\mathrm{N}$ and $\mathrm{Np}$ atoms.

In Figs. 4(a) and 4(b), we show the calculated real and imaginary parts of the off-diagonal optical conductivity $\sigma_{x y}$ multiplied by $\omega$, respectively. In these figures, solid lines represent the results of the FFLCAO calculations, and dotted lines, the results of the FFMB calculations. The agreement is again found to be satisfactory. The non-zero off-diagonal optical conductivity is the direct consequence of spin-orbit coupling, and thus, it is large in materials with heavy atoms. The large off-diagonal optical conductivity of $\mathrm{NpN}$ is due to the large spin-orbit coupling in Np atoms. In fact, the off-diagonal optical conductivity of US is almost of the same magnitude because of the large spin-orbit coupling in U atoms. On the other hand, it is found that the off-diagonal optical conductivity of $\mathrm{NpN}$ is several times larger than that of $\mathrm{Fe}$. A broad peak is also found in the imaginary part around 3-6 eV. A corresponding structure is found in the real part around 3-6 eV. Analyzing the origin of transitions, we found that the two transitions producing the broad peak are the same as those responsible for the broad peak in the real part of the diagonal optical conductivity. We found that the contributions of the two transitions are almost of the same magnitude. This is not surprising because the $\mathrm{N}-2 p$ orbitals are strongly hybridized with the Np-5f orbitals, and thus, the transition can be 
enhanced by the large spin-orbit coupling to a considerable extent.

Since the optical conductivity tensor is not a quantity measured directly in experiments, we here show the reflectivity and Kerr spectra. In Figs. 5(a) and 5(b), we show the reflectivity spectra for the left and right circular polarizations, respectively. Also, in Figs. 6(a) and 6(b), we show the Kerr rotation and ellipticity spectra, respectively. In these figures, solid lines represent the results of the FFLCAO calculations, and dotted lines, the results of the FFMB calculations. It is found that the Kerr rotation of $\mathrm{NpN}$ is considerably large. The maximum rotation angle is about 2 degrees. This is comparable to that of US, about 3 degrees. ${ }^{13,14,17)}$ Also, the Kerr rotation spectra change its sign at about $5 \mathrm{eV}$, as is also common to both materials. The large Kerr rotation angle is due to the large spin-orbit coupling in Np atoms. This is evident when comparing with the small Kerr rotation angle of Fe, for which the maximum rotation angle is about 0.6 degrees. ${ }^{32,44)}$

Finally, we show the results of the calculations of the total absorption and XMCD spectra of the $M_{4,5}$ edges of Np. In Figs. 6(a)-6(c), we show the total absorption, the XMCD of the $M_{5}$ edge, and the XMCD of the $M_{4}$ edge, respectively. In the figures, solid lines represent the results of the FFLCAO calculations, and dotted lines, the results of the FFMB calculations. These results are in good agreement with each other and overlap almost perfectly. The $M_{4}$ and $M_{5}$ edges originate in the transitions $3 d_{3 / 2} \rightarrow 5 f$ and $3 d_{5 / 2} \rightarrow 5 f$, respectively. Although there are no experimental results available, we may compare the peak energies of the total absorption with those measured in another Np compound. ${ }^{45)}$ The calculated peak energy of the $M_{4}$ edge is $3.788 \mathrm{keV}$ and the measured one is $3.845 \mathrm{keV}$; the error is $-1.5 \%$. Also, the calculated peak energy of the $M_{5}$ edge is $3.600 \mathrm{keV}$ and the measured one is $3.664 \mathrm{keV}$; the error is $-1.7 \%$. Accordingly, the calculated energy difference between the two peaks is $0.188 \mathrm{keV}$ and the measured one is $0.181 \mathrm{keV}$; the error is $3.9 \%$. The shape of the calculated XMCD spectra of the $M_{4}$ edge is symmetric while that of the $M_{5}$ edge is strongly asymmetric. Furthermore, we calculate the ratio of the integrated intensity of the $M_{5}$ edge to that of the $M_{4}$ edge and find it to be 0.17 ; we confirmed that this result is almost independent of the choice of the lifetime parameter. The integrated intensity of the $M_{5}$ edge is smaller than that of the $M_{4}$ edge by about one order of magnitude. This is also found in US, for which the ratio is $0.13 \pm 0.03 .{ }^{46)}$ We also estimate the orbital magnetic moment from the total absorption and XMCD spectra using the XMCD sum rule. ${ }^{28,29)}$

$$
\frac{\int_{M_{4}+M_{5}} \Delta \mu \mathrm{d} \omega}{\int_{M_{4}+M_{5}}\left(\mu_{\mathrm{tot}}+\mu_{z}\right) \mathrm{d} \omega}=\frac{1}{3 n_{\mathrm{h}}} M_{\mathrm{orb}}
$$

In this equation, $n_{\mathrm{h}}$ represents the number of holes in the Np-5f orbitals; we used $n_{\mathrm{h}}=9.8$ obtained by the Mulliken population analysis of the results of the FFLCAO calculations. ${ }^{47)}$ It is also confirmed that the relation $\mu_{z}=\mu_{\text {tot }} / 2$ holds approximately. We find that the sum rule gives $M_{\mathrm{orb}}=1.71 \mu_{\mathrm{B}}$, which is substantially smaller than that given in Table II, 2.55 
$\mu_{\mathrm{B}}$. The large difference implies that there is a lack of internal consistency in calculating the orbital magnetic moment using the two methods. The disagreement is disappointing, because the XMCD sum rule for the orbital magnetic moment has been shown to work well for the $3 d$ transition metals and the method is expected to be promising in the determination of the orbital magnetic moment experimentally. ${ }^{48)}$ Although the origin of the disagreement is not clear, some approximations used in deriving the sum rule might not be applicable for actinide atoms; for example, the replacement of the matrix elements of the Dirac matrices with those of the momentum operator might result in such an error because this approximation is not very good even for Fe when calculating physical quantities sensitive to spin-orbit coupling. ${ }^{32}$ ) Furthermore, as shown in a recent experimental study of the XMCD spectrum of the $N_{4,5}$ edges of uranium calcogenides, the applicability of the XMCD sum rule might be limited. ${ }^{49)}$ Thus, a more careful examination of the XMCD sum rule should be performed both theoretically and experimentally in the future.

\section{Conclusions}

We have studied the structural, electronic, magnetic, optical, and magneto-optical properties of $\mathrm{NpN}$ in detail using the fully relativistic full-potential calculations based on the density functional theory within the LSDA. We employed two methods, namely, the FFLCAO and FFMB methods, to examine the reliability of the results with respect to the quality of basis sets, and found that the results obtained by the FFLCAO and FFMB methods are in good agreement with each other. The optimized lattice constant and calculated cohesive energy are in reasonable agreement with the experimental results within an acceptable error known for the LSDA. It was also found that the magnitude of the calculated orbital magnetic moment is larger than that of the calculated spin magnetic moment and the calculated total magnetic moment is considerably smaller than the experimental one. Furthermore, we have successfully reproduced the positive sign of the EFG at the Np nuclei. The positive EFG is in striking contrast to the negative EFG in a $\mathrm{Np}^{3+}$ free ion, indicating the importance of the interplay between spin-orbit coupling and covalent bonding. The calculated band structure shows large splittings induced by spin polarization and spin-orbit coupling, suggesting that both the effects are indispensable for understanding the electronic properties of this material. Moreover, using the calculated optical conductivity tensor, we have studied the reflectivity and Kerr spectra. As a result, we found that the calculated Kerr rotation spectrum shows a maximum value of about 2 degrees, which is comparable to those observed in uranium calcogenides. Finally, also using the calculated optical conductivity tensor, we have studied the XMCD spectra and found that the orbital magnetic moment estimated using the XMCD sum rule is considerably smaller than that calculated by the conventional method, although the origin of disagreement is not clear. 


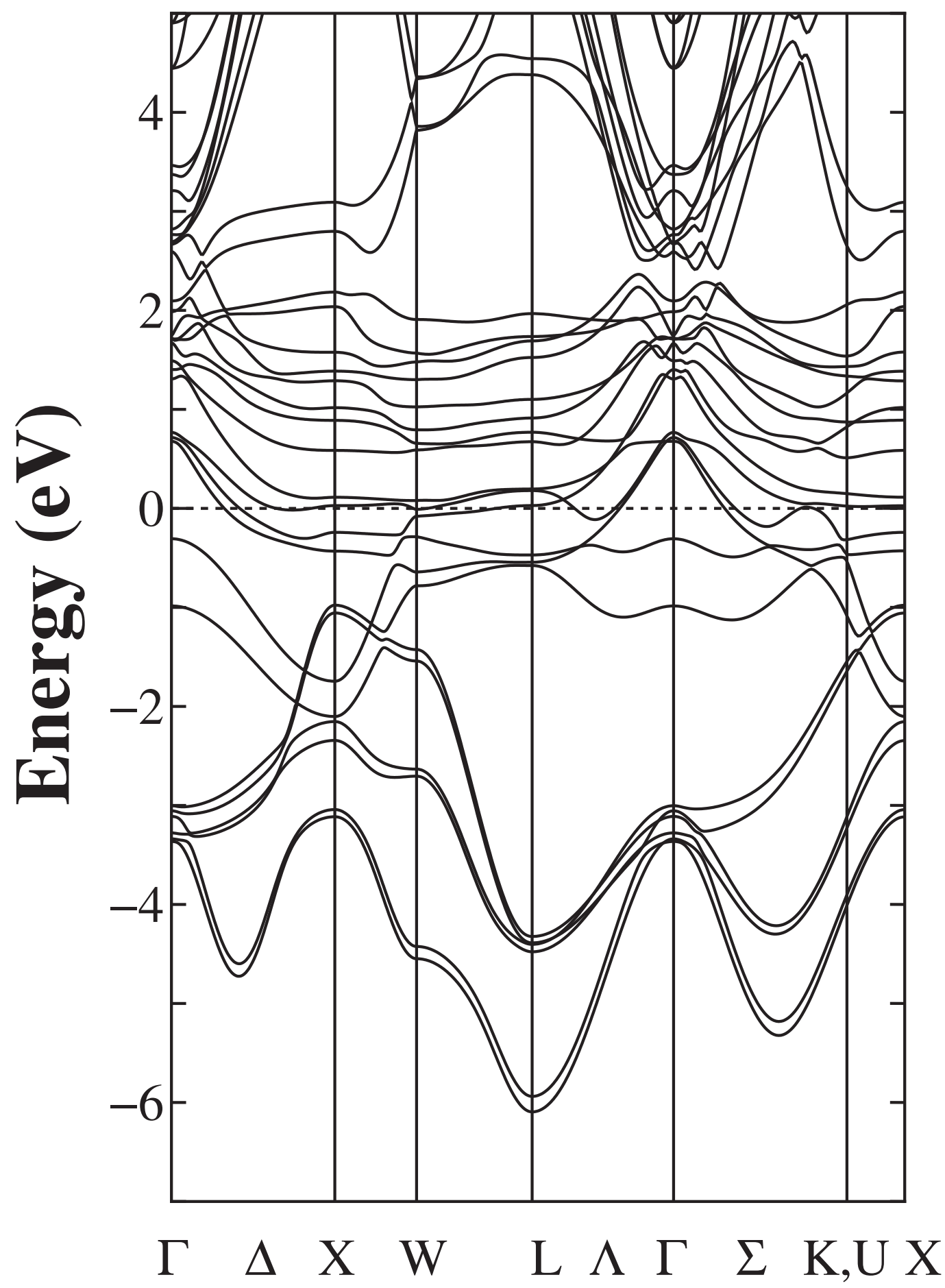

Fig. 1. Band structure of NpN calculated using fully relativistic full-potential LCAO method. The dotted line represents the Fermi level. 


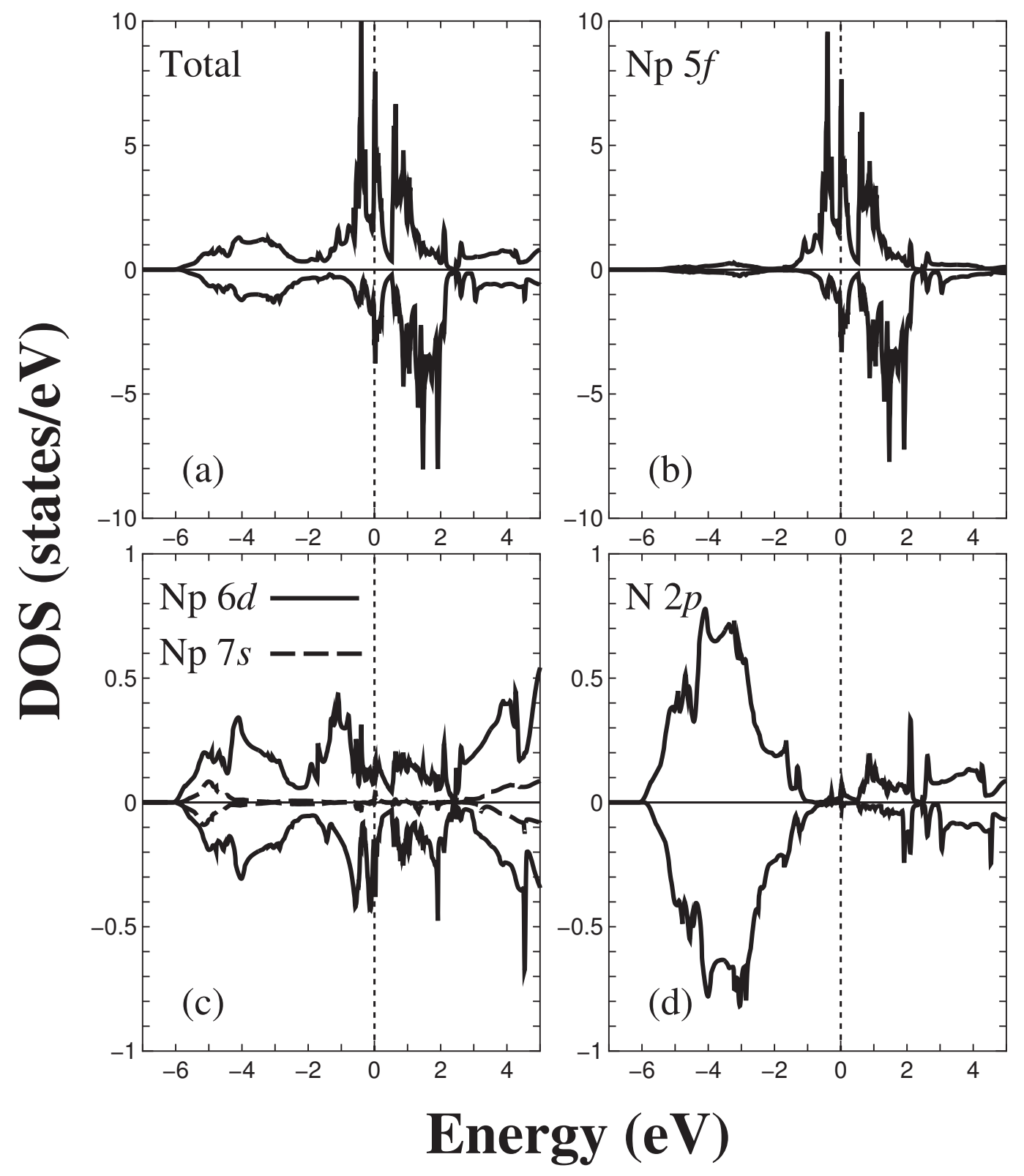

Fig. 2. Spin-projected density of states (SPDOS) per formula unit of NpN calculated using fully relativistic full-potential LCAO method: (a) total SPDOS, (b) partial SPDOS for Np-5 $f$ orbitals, (c) partial SPDOS for Np-6d and Np-7s orbitals, and (d) partial SPDOS for N-2p orbitals. Upper panels are for up-spin electrons and lower panels are for down-spin electrons. The dotted line represents the Fermi level. 


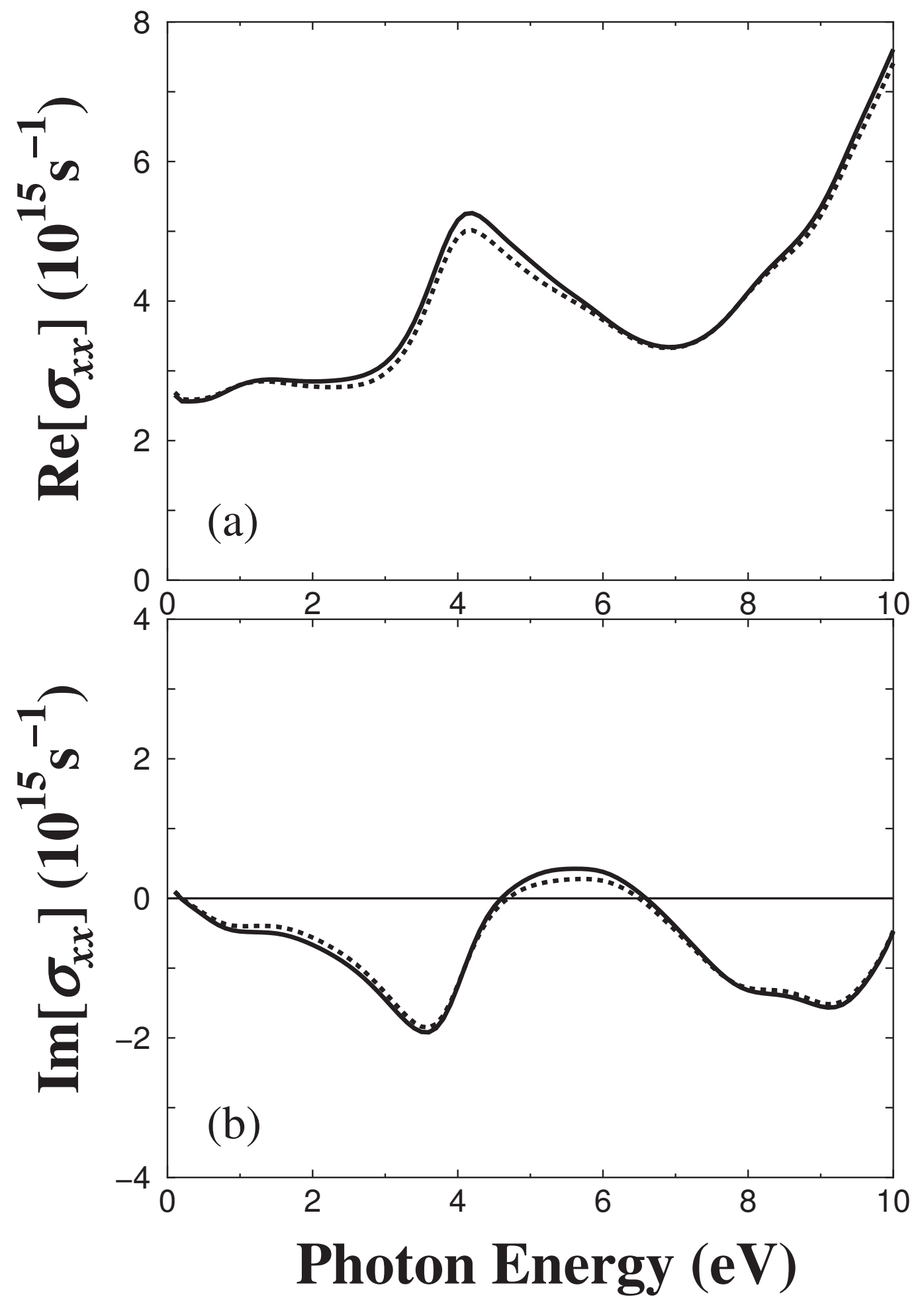

Fig. 3. Diagonal optical conductivity: (a) real part and (b) imaginary part. Only the interband contribution is considered. Solid lines represent the results of the fully relativistic full-potential LCAO calculations, and dotted lines, the results of the fully relativistic full-potential mixed-basis calculations. The lifetime parameter of $0.5 \mathrm{eV}$ is used. 


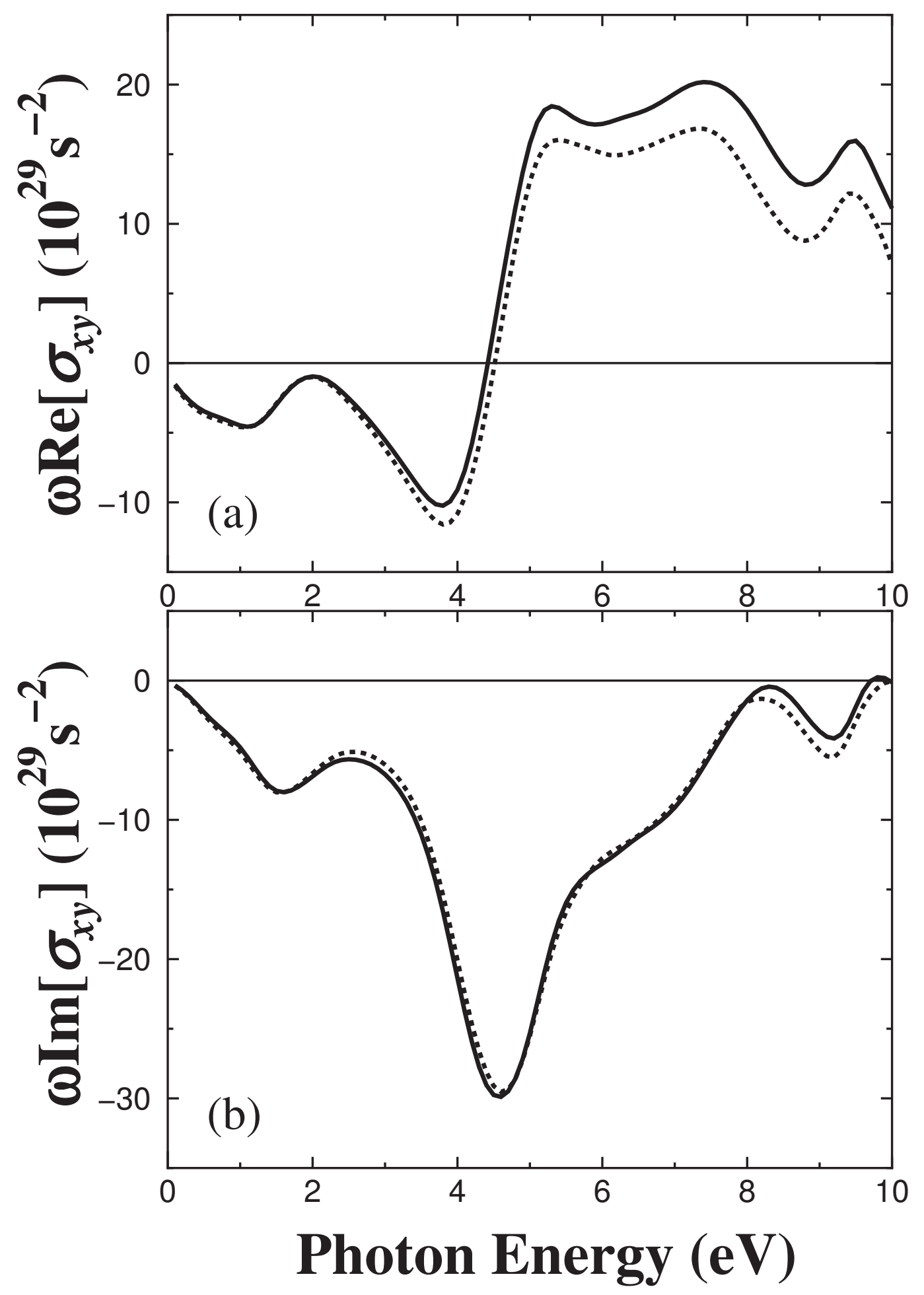

Fig. 4. Off-diagonal optical conductivity multiplied by $\omega$ : (a) real part and (b) imaginary part. Solid lines represent the results of the fully relativistic full-potential LCAO calculations, and dotted lines, the results of the fully relativistic full-potential mixed-basis calculations. The lifetime parameter of $0.5 \mathrm{eV}$ is used. 


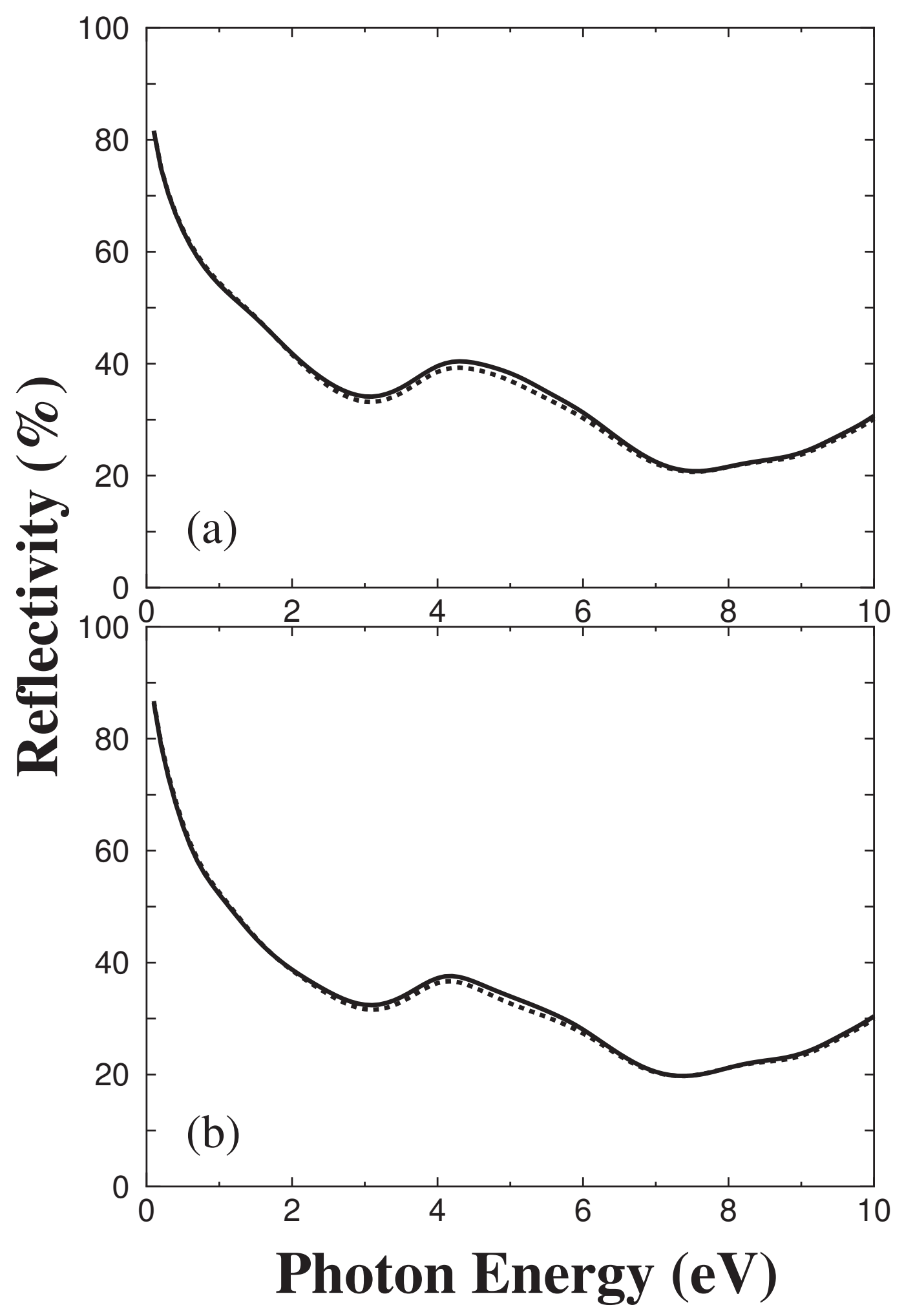

Fig. 5. Reflectivity spectra of $\mathrm{NpN}$ for (a) left circular and (b) right circular polarizations. Solid lines represent the results of the fully relativistic full-potential LCAO calculations and, dotted lines, the results of the fully relativistic full-potential mixed-basis calculations. The lifetime parameter of $0.5 \mathrm{eV}$ is used. 


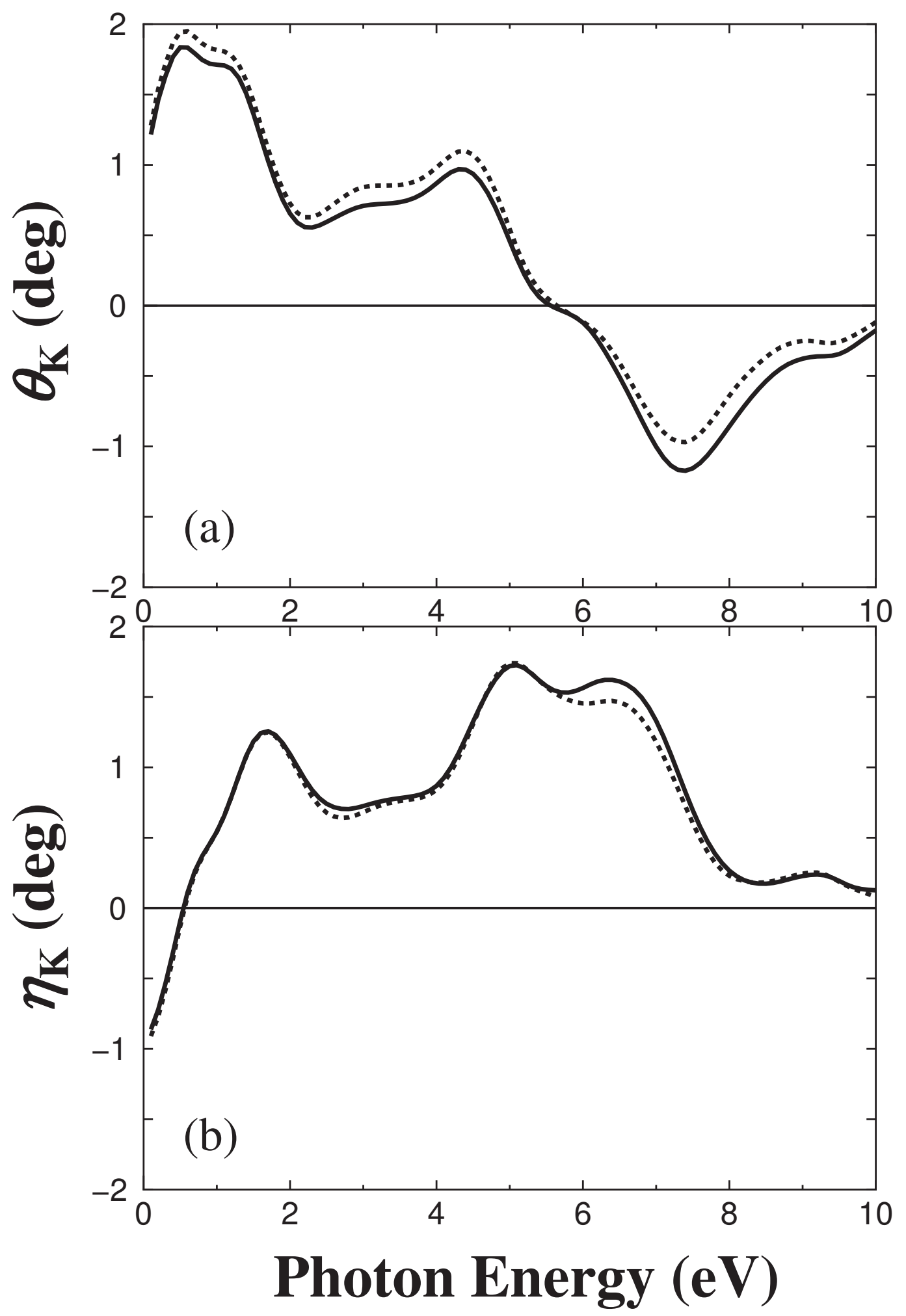

Fig. 6. Kerr spectra of $\mathrm{NpN}$ : (a) Kerr rotation angle and (b) Kerr ellipticity. Solid lines represent the results of the fully relativistic full-potential LCAO calculations, and dotted lines, the results of the fully relativistic full-potential mixed-basis calculations. The lifetime parameter of $0.5 \mathrm{eV}$ is used. 

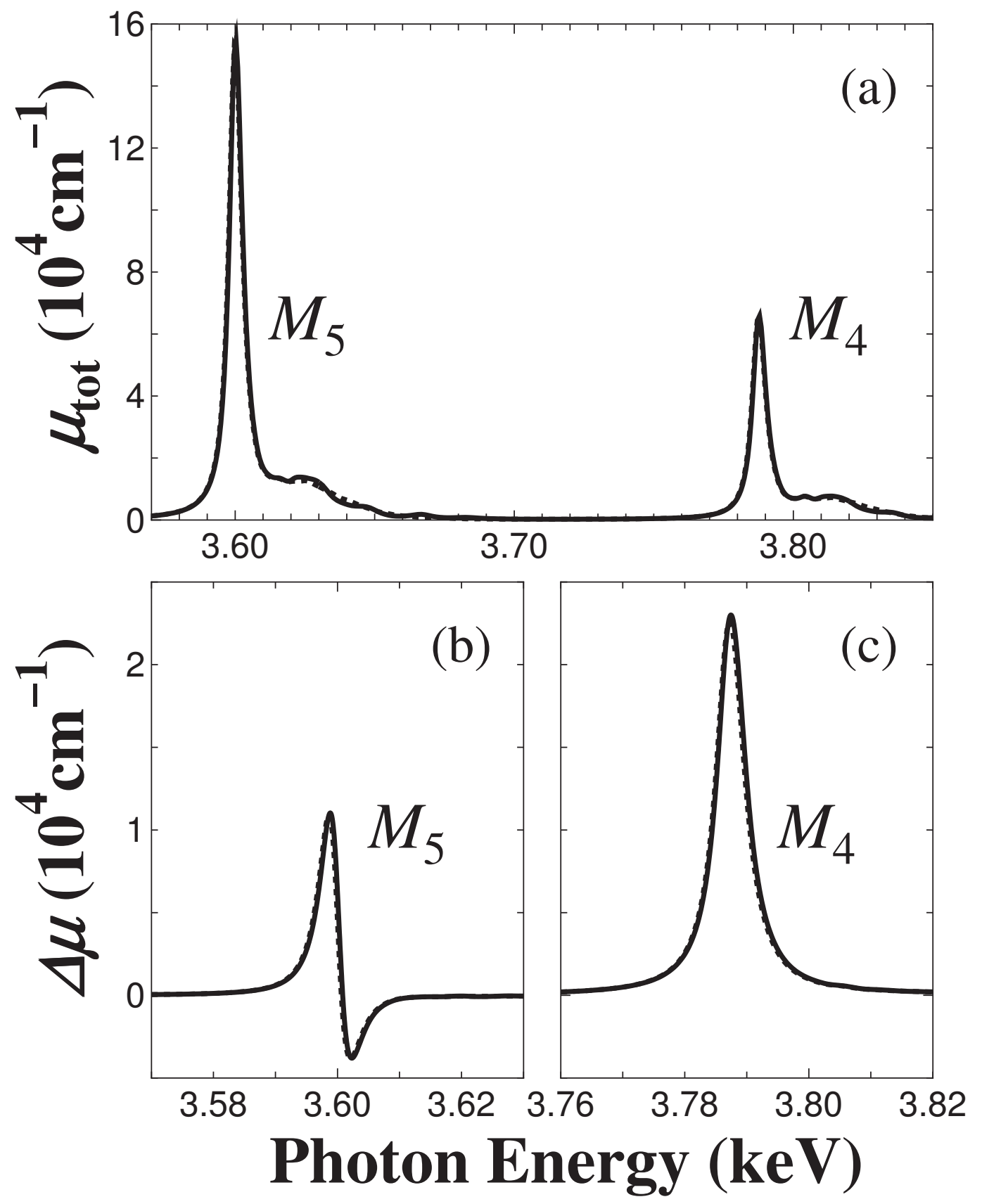

Fig. 7. Total absorption and XMCD spectra of $M_{4,5}$ edges of $\mathrm{NpN}$ : (a) total absorption, (b) XMCD of $M_{5}$ edge, and (c) XMCD of $M_{4}$ edge. Solid lines represent the results of the fully relativistic full-potential LCAO calculations, and dotted lines, the results of the fully relativistic full-potential mixed-basis calculations. These results are in good agreement with each other and overlap almost perfectly. The lifetime parameter of $2.5 \mathrm{eV}$ is used. 


\section{References}

1) Handbook on the Physics and Chemistry of the Actinides, ed. A. J. Freeman and G. H. Lander (North-Holland, Amsterdam, 1985).

2) A. T. Aldred, B. D. Dunlap, A. R. Harvey, G. H. Lander, and M. H. Mueller: Phys. Rev. B 9 (1974) 3766.

3) M. B. Brodsky: Rep. Prog. Phys. 41 (1978) 103.

4) M. S. S. Brooks: J. Magn. Magn. Mater. 29 (1982) 257.

5) M. S. S. Brooks and P. J. Kelly: Phys. Rev. Lett. 51 (1983) 1708.

6) M. S. S. Brooks: J. Phys. F: Phys. 14 (1984) 639.

7) M. S. S. Brooks: J. Phys. F: Phys. 14 (1984) 653.

8) M. S. S. Brooks: J. Phys. F: Phys. 14 (1984) 857.

9) M. S. S. Brooks: Physica 130B (1985) 6.

10) M. S. S. Brooks, B. Johansson, O. Eriksson, and H. L. Skriver: Physica 144B (1986) 1.

11) W. Reim and J. Schoenes: in Ferromagnetic Materials, ed. K. H. J. Buschow and E. P. Wohlfarth (Elsevier Science Publishers B.V., Amsterdam, 1990) Vol. 5, p. 133.

12) S. V. Halilov and E. T. Kulatov: J. Phys.: Condens. Matter 3 (1991) 6363.

13) M. S. S. Brooks, T. Gasche, and B. Johansson: J. Phys. Chem. Solids 56 (1995) 1491.

14) T. Kraft, P. M. Oppeneer, V. N. Antonov, and H. Eschrig: Phys. Rev. B 52 (1995) 3561.

15) J. Trygg, J. M. Wills, M. S. S. Brooks, B. Johansson, and O. Eriksson: Phys. Rev. B 52 (1995) 2496.

16) H. Yamagami: J. Phys. Soc. Jpn. 67 (1998) 3176.

17) V. N. Antonov, A. N. Yaresko, A. Ya. Perlov, V. V. Nemoshkalenko, P. M. Oppeneer, and H. Eschrig: Low Temp. Phys. 25 (1999) 387.

18) K. Nakajima, Y. Arai, and Y. Suzuki: J. Nucl. Mater. 275 (1999) 332.

19) H. Yamagami: Phys. Rev. B 61 (2000) 6246.

20) T. Shishidou: Phys. Rev. B 62 (2000) 11747.

21) P. M. Oppeneer and T. Kraft: Phys. Rev. B 61 (2000) 12825.

22) A. Svane, L. Petit, W. M. Temmerman, and Z. Szotek: Phys. Rev. B 66 (2002) 085110.

23) V. N. Antonov, B. N. Harmon, O. V. Andryushchenko, L. V. Bekenev, and A. N. Yaresko: Low Temp. Phys. 30 (2004) 305.

$24)$ D. B. Ghosh, S. K. De, P. M. Oppeneer, and M. S. S. Brooks: Phys. Rev. B 72 (2005) 115123.

25) D. Sedmidubský, R. J. M. Konings, and P. Novák: J. Nucl. Mater. 344 (2005) 40.

26) P. F. Weck, E. Kim, N. Balakrishnan, F. Poineau, C. B. Yeamans, and K. R. Czerwinski: Chem. Phys. Lett. 443 (2007) 82.

27) R. Atta-Fynn and A. K. Ray: Phys. Rev. B 76 (2007) 115101.

$28)$ B. T. Thole, P. Carra, F. Sette, and G. van der Laan: Phys. Rev. Lett. 68 (1992) 1943.

29) P. Carra, B. T. Thole, M. Altarelli, and X. Wang: Phys. Rev. Lett. 70 (1993) 694.

30) C. T. A. M. de Laat, A. Taal, W. Duinker, J. Konijn, J. F. M. DAchard Van Enschut, P. David, J. Hartfiel, H. Janszen, T. Mayer-Kuckuk, R. von Mutius, C. Piller, L. A. Schaller, L. Schellenberg, T. Kroguiski, C. Perirjean, H. W. Reist, and W. Müller: Phys. Lett. B 189 (1987) 7.

31) S. Suzuki and K. Nakao: J. Phys. Soc. Jpn. 68 (1999) 1982.

32) M.-F. Li, T. Ariizumi, and S. Suzuki: J. Phys. Soc. Jpn. 76 (2007) 054702. 
33) H. Ebert and R. Zeller: Phys. Rev. B 42 (1990) 2744.

34) E. Arola, P. Strange, and B. L. Gyorffy: Phys. Rev. B 55 (1997) 472.

35) D. M. Ceperley and B. J. Alder: Phys. Rev. Lett. 45 (1980) 566.

36) J. Perdew and Y. Wang: Phys. Rev. B 23 (1981) 5048.

37) H. J. Monkhorst and J. D. Pack: Phys. Rev. B 13 (1976) 5188.

38) L.-K. Hua and Y. Wang: Applications of Number Theory to Numerical Analysis (Springer-Verlag, Berlin, 1981).

39) F. D. Murnaghan: Proc. Natl. Acad. Sci. USA 30 (1944) 244.

40) M. Singh, C. S. Wang, and J. Callaway: Phys. Rev. B 11 (1975) 287.

41) C. P. Slichter: Principles of Magnetic Resonance (Springer-Verlag, Berlin, 1990).

42) S. Suzuki and K. Nakao: J. Phys. Soc. Jpn. 66 (1997) 3881.

$43)$ M. D. Jones, J. C. Boettger, and R. C. Albers: Phys. Rev. B 61 (2000) 4644.

44) P. M. Oppeneer, T. Maurer, J. Sticht, and J. Kübler: Phys. Rev. B 45 (1992) 10924.

45) S. B. Wilkins, J. A. Paixão, R. Caciuffo, P. Javorsky, F. Wastin, J. Rebizant, C. Detlefs, N. Bernhoeft, P. Santini, and G. H. Lander: Phys. Rev. B 70 (2004) 214402.

46) S. P. Collins, D. Laundy, C. C. Tang, and G. van der Laan: J. Phys.: Condens. Matter 7 (1995) 9325.

47) R. S. Mulliken: J. Chem. Phys. 23 (1995) 1833.

48) R. Wu, D. Wang, and A. J. Freeman: Phys. Rev. Lett. 71 (1993) 3581.

49) T. Okane, Y. Takeda, J. Okamoto, K. Mamiya, T. Ohkochi, S. Fujimori, Y. Saitoh, H. Yamagami, A. Fujimori, A. Ochiai, and A. Tanaka: J. Phys. Soc. Jpn. 77 (2008) 02476. 